\title{
Logic, rationality and interaction (LORI-5): introduction to the special issue
}

\author{
Wiebe van der Hoek ${ }^{1}$. Wesley H. Holiday ${ }^{2}$. \\ Wen-fang Wang ${ }^{3}$
}

This special issue of Synthese contains revised and extended versions of selected papers from LORI-5, the 5th International Workshop on Logic, Rationality and Interaction. LORI-5 was held during October 28-31, 2015, in Taipei, Taiwan, and hosted by the Department of Philosophy of National Taiwan University and the Institute of Philosophy of Mind and Cognition of National Yang-Ming University. The hallmark of the LORI series is its broad topical coverage, putting logic into contact with disciplines as diverse as game theory and decision theory, epistemology and philosophy of language, linguistics, computer science, and artificial intelligence. In the current special issue, the reader will find several mainstays of the LORI series: modal and epistemic logics; theories of decision making, strategic reasoning, and information aggregation; and connections between logic and natural language.

Out of 62 original submissions to LORI-5, 32 full papers were selected for presentation based on reviews by at least two-and on average three-members of the LORI-5 program committee. After the workshop, the authors of 20 of the full papers were invited to submit extended versions of their papers to be considered for either the present special issue or a parallel special issue of the Journal of Logic and Computation. Each of the nine papers considered for this special issue underwent review by two

\footnotetext{
$凶 \quad$ Wiebe van der Hoek wiebe@liverpool.ac.uk

Wesley H. Holiday wesholliday@berkeley.edu

Wen-fang Wang wenfwang88@gmail.com

1 University of Liverpool, Liverpool, UK

2 University of California, Berkeley, CA, USA

3 National Yang-Ming University, Taipei, Taiwan
} 
or three referees, leading to at least one-and in some cases two or three-rounds of revision. The final products are the eight papers collected in this issue. As an overview of its contents, below we provide a brief summary of each paper (in alphabetical order by author's last name).

In 'A Simpler and More Realistic Subjective Decision Theory', Haim Gaifman and Yang Liu show how to relax two assumptions of Leonard Savage's influential framework for decision theory. The first assumption, about which Savage himself had reservations, is that the algebra of events over which an agent has probabilities is closed under all countable intersections and unions. The second assumption, which is known to be counterintuitive, is the so-called constant act assumption, stating that for every possible consequence there is an act that has that consequence in every state of the world. The authors show how to weaken both of these assumptions. They argue that doing so makes the resulting decision theory applicable to more realistic rational agents, e.g., agents who are not able to assess the utilities of physically impossible scenarios, as the idealized agents in Savage's framework must be able to.

The contribution of Konstantinos Georgatos, 'Geodesic Merging', is on belief merging, which is an operation that combines possibly inconsistent propositions into a consistent one. Studies of belief merging contribute to the logical foundations of research fields as diverse as database fusion, networking, and judgement aggregation, amongst others. This particular paper utilises geodesic semantics for merging, a semantics based on the shortest path in a graph. Three binary merging operators of propositions are introduced to this end, defined on the graph of their valuations. These operators are characterised with a finite set of postulates. The author then considers a revision operator defined in the language of pairs of propositions: this extension enables the expression of all merging operators through a set of revision postulates.

In 'Studying strategies and types of players: Experiments, logics and cognitive models', Sujata Ghosh, Rineke Verbrugge, Tamoghna Halder and Khyati Sharma present new experimental research on human subjects playing turn-based games. The authors then propose a logical language for expressing different kinds of strategies that people applied in the game-theoretic experiment, as well as algorithms based on the cognitive architecture Adaptive Control of Thought-Rational (ACT-R), implementing strategies expressed in their logical formalism. More specifically, they investigate whether subjects in such games are playing a forward reasoning strategy or a backward reasoning strategy and account for the results of the experiment by classifying the players into different strategic types. Thus, this article provides a three-way interaction between experiments, logic and cognitive modeling so as to bring out a shared perspective across these diverse areas.

In 'Informational Dynamics of Epistemic Possibility Modals', Peter Hawke and Shane Steinert-Threlkeld advance the thesis that the primary function of an assertion is to express the presence/absence of certain beliefs in the doxastic state of the speaker. The authors prove in a logical setting that an arbitrary assertion involving possibly nested occurrences of 'might' always (1) expresses a finite set of beliefs and socalled abeliefs (lack of beliefs) and (2) determines a corresponding doxastic update operation. Leaving a comprehensive philosophical and linguistic defense aside, they also explore some technical aspects of their proposed logical framework in detail, providing, among other results, a complete logic of assertability and reduction axioms 
for the novel update operations. Finally, they compare and contrast their work with related proposals.

In 'Knowledge, Belief, Normality, and Introspection', Dominik Klein, Olivier Roy and Norbert Gratzl take as their starting point a logic for reasoning about knowledge and belief proposed by Robert Stalnaker. A fascinating aspect of this logic is that it makes belief definable in terms of knowledge: the equivalence $B \varphi \leftrightarrow \neg K \neg K \varphi$ is a theorem of the logic. The authors depart from Stalnaker's logic by dropping a philosophically contentious axiom of his system, namely the "positive introspection" axiom for knowledge, $K \varphi \rightarrow K K \varphi$, while retaining the definition of belief by $B \varphi \leftrightarrow$ $\neg K \neg K \varphi$. Their paper shows that dropping positive introspection brings surprising consequences for the logic of belief in the resulting system.

The title 'On the Expressive Power of First-Order Modal Logic with TwoDimensional Operators' by Alexander Kocurek is well-chosen: in the paper, Kocurek gives a formal analysis of the relative expressive power of first-order modal logic with quantifiers ranging over (possible and/or actual) existents that is enriched with twodimensional operators ('actually' being an example of such an operator). Examples of phrases that cannot be expressed in first-order modal logic are 'There could have been things other than there actually are' and 'Everyone who's actually rich could have been poor'. Until recently, the proofs of inexpressibility claims for various extensions of first-order modal logic have been informal or ad hoc, and some have relied on particular features of the formulation of first-order modal logic in question, such as the universality of the accessibility relation. The author's contribution is the development of a modular notion of bisimulation in terms of back-and-forth games to characterise the expressive power of extensions of first-order modal logic with two-dimensional operators. He then presents a variety of inexpressibility results using this notion and generalises his results to temporal logics and higher-dimensional logics, showing, for instance, that each $n$-dimensional logic is expressively weaker than the corresponding $n+1$-dimensional logic, a result that was conjectured but not proven in the literature.

Yanjing Wang proposes, in 'A Logic of Goal-Directed Knowing How', a singleagent modal logic based on ideas from linguistics, philosophy, modal logic, and automated planning in artificial intelligence. The basic language is defined to express statements of the form 'I know how to guarantee $\varphi$ given $\psi$ '. Rather than using standard epistemic models, the semantics of this language is based on labeled transition systems, which represent the agent's knowledge of their own abilities. A sound and complete proof system is provided, and the logic is shown to be decidable. The logical language is then enriched to enable reasoning about situations where the agent knows how to achieve a goal while at the same time maintaining some side conditions.

Finally, in 'Three-Valued Semantic Pluralism: A Defense of A Three-Valued Solution to the Sorites Paradox', Wen-fang Wang addresses the Sorites paradox. Central in the paper is a first-order language that deals with vague predicates and identity. The author proposes a three-valued semantics for this language, where the basic assumption is that a vague language allows for more than one interpretation. Unlike more traditional three-valued approaches to vague languages, three-valued semantic pluralism can accommodate higher-order vagueness and the phenomenon of penumbral connection. The author then claims that his semantics follows naturally from a restricted 
version of the Tolerance Principle, which in turn is well-motivated by considerations about how we learn, teach, and use vague predicates.

We hope that these papers will not only advance the state of the art on their respective topics, but also provide for those unable to attend LORI-5 a sense of the stimulating diversity of the workshop.

Acknowledgements We thank Otávio Bueno for his patience and support in overseeing this issue. 